\title{
Review of: "Magnetic particle imaging of magnetotactic bacteria as living contrast agents is improved by altering magnetosome structures"
}

Daniel Acosta-Avalos

Potential competing interests: The author(s) declared that no potential competing interests exist.

The preprint "Magnetic particle imaging of magnetotactic bacteria as living contrast agents is improved by altering magnetosome structures" is an interesting study about using whole magnetotactic bacteria for magnetic particle imaging in whole animals. The authors used natural samples (wild type) and samples with the mamJ protein deleted (DeltamamJ) of the well known Magnetospirillum gryphiswaldense strain MSR1. The authors observed that the magnetotactic bacteria MSRDeltamamJ is better for magnetic particle imaging in whole animals and excised organs. The text is well written and easy to read.

As far as I know, the presente study is the first to show an application of live magnetotactic bacteria not associated to magnetotaxis but to the fabrication of magnetosomes aggregated in clusters. These results are the first of a promising application of magnetotactic bacteria as contrast for a biomedical imaging technique.

I have some minor comments, to improve the manuscript and the study:

1. In page 3 you wrote that "magnetotactic bacteria are organisms with the unique ability to biomineralize iron into cuboctahedral magnetite crystals". That is partially true. Magnetotactic bacteria are able to biomineralize magnetite in other crystalline habits and also biomineralize greigite (Islam, Peng and Ali; J Basic Microbiol. 58: 378-389. DOI:10.1002/jobm.201700383).

2. Can your results change if you use greigite magnetosomes or magnetosomes different from the cuboctahedral habits?

3. In page 4 you wrote that "genetic deletions or knockouts within the magnetosome island have been shown to affect magnetosome assembly and structure" and that "deletions of mamK and mamJ genes.... affect the overall magnetosome structures". What you mean with magnetosome structure? Magnetosome is an organelle composed by membrane that biomineralize a magnetic nanoparticle. In normal magnetotactic bacteria magnetosomes are organized in chains (there are some natural magnetotactic bacteria that do not show magnetosome chains). I read references 25, 26, 27, 28 and 29 that you cite to that respect, but in all of them they show the effect of those genes in the magnetosome chain formation and not in the magnetosome structure. Please, be more specific in this part of the manuscript and even in your paper title.

4. In figure 3, the absence of MPI signal in WT sample can be due to a very weak signal from the sample, as happens in Figure 2? Perhaps if you change the scale appears a weak signal. 
5. In figures $3 b$ and $3 c$, can you explain the units used for radiance?

6. Just as a curiosity, in page 9 you report the percent injected dose, as determined by MPI signal, as $42+-$ $28 \%$ at $5 \mathrm{~m}, 30+-1 \%$ at $1 \mathrm{~h}$ and $62+-20 \%$ at $24 \mathrm{~h}$. Why the standard deviation is so small at $1 \mathrm{~h}$ compared with the other measurement times?

7. In figure $4 b$, why at time 0 you report a MPI signal in the liver? What is the origin of that signal?

8. Figures $4 a$ and $4 c$ give different pictures from the same phenomenum, but is confusing. MPI signal shows that bacteria concentrate in the liver with time, but BLI shows that bacteria distribute in all the body after 5 mins. Why is not observed the same with MPI signal at 5 mins?

Those were my questions. I hope they will be useful and I wish you success in your future studies appying magnetotactic bacteria into biomedical areas. 